\title{
Hepatitis B virus $X$ protein modifies invasion, proliferation and the inflammatory response in an HTR-8/SVneo cell model
}

\author{
HONG CUI, QIU-LING LI, JING CHEN, QUAN NA and CAI-XIA LIU \\ Department of Obstetrics and Gynecology, Shengjing Hospital of China Medical University, \\ Shenyang, Liaoning 110004, P.R. China
}

Received May 11, 2015; Accepted July 6, 2015

DOI: $10.3892 /$ or.2015.4172

\begin{abstract}
Mother-to-infant transmission of hepatitis $\mathrm{B}$ virus (HBV) can occur as an intrauterine, intrapartum or postpartum infection. In the present study, we induced a multifunctional viral regulator of $\mathrm{HBV}$ gene products, $\mathrm{HBx}$, and its different fragments to overexpress in a trophoblast cell line, HTR-8/SVneo. We then identified the biological effects of HBx on HTR-8/SVneo cells. Our results indicated that HBx inhibited apoptosis and induced invasion as detected using Annexin V/propidium iodide (PI) double staining and Transwell assay, respectively. Furthermore, we carried out western blot analysis to analyze the possible related signaling pathway. We confirmed that $\mathrm{HBx}$ and its different fragments can activate the Smad signaling pathway, accompanied by downregulation of E-cadherin, and upregulation of vimentin and $\mathrm{N}$-cadherin. TGF- $\beta 1$ was used as a control to activate the Smad signaling pathway in HTR-8/SVneo cells. HBx activated the Smad signaling pathway in the HTR-8/SVneo cells. After the signaling pathway was activated, reduced apoptosis, higher invasive ability and enhanced inflammatory response were observed in the HTR-8/SVneo cells.
\end{abstract}

\section{Introduction}

Hepatitis B virus (HBV) infection is a major global health issue, and more than 350 million individuals are infected by HBV worldwide (1). Approximately $10-15 \%$ of pregnant women in China are carriers of the HBV surface antigen (HBsAg) and HBV e antigen (HBeAg) and 5-15\% of their babies are infected by intrauterine transmission $(2,3)$. The mechanism of intrauterine HBV infection is not completely understood; transplacental leakage of maternal blood has been suggested as a possible mechanism (4). Trophoblasts, special-

Correspondence to: Professor Cai-Xia Liu, Department of Obstetrics and Gynecology, Shengjing Hospital of China Medical University, Heping, Shenyang, Liaoning 110004, P.R. China

E-mail: liucx176@163.com

Key words: Hepatitis $\mathrm{B}$ virus $\mathrm{X}$ protein, trophoblast, apoptosis, invasion, Smad signaling ized cells of the placenta, mediate the contact between two genetically different individuals, the embryo and the mother, by establishing a transient embryonic organ, the placenta (5). Impaired trophoblast function may result in a range of adverse pregnancy outcomes, such as spontaneous abortion, intrauterine infection and stillbirth (6).

Among the HBV gene products, the hepatitis $\mathrm{B}$ virus $\mathrm{X}$ protein $(\mathrm{HBx})$ is a multifunctional viral regulator and is considered as one of the most important determinants involved in viral pathogenesis and carcinogenesis (7). HBx, which consists of 154 amino acids, contains four regions important for transcriptional regulation, cell cycle control, cell adhesion and modulation of cytoplasmic signal transduction pathways (8). Different regions of $\mathrm{HBx}$ may play different roles in the pathological process. For example, $H B x \Delta 127$ (deletion from 382 to $401 \mathrm{bp}$ ) was able to upregulate transcriptional activities of nuclear factor- $\kappa \mathrm{B}$, survivin and human telomerase reverse transcriptase, as well as the expression levels of c-Myc and proliferating cell nuclear antigen in hepatoma cells (9).

To our knowledge, the role of $\mathrm{HBx}$ in trophoblasts remains unclear. In the present study, we constructed two HBx mutants, including $\mathrm{N}$-terminal and $\mathrm{C}$-terminal mutants, and analyzed the biological activities of HTR-8/SVneo cells with those truncated mutants of the HBx gene. Finally, we identified the underlying mechanism involving the promotion of cell growth and invasion mediated by the HBx mutants.

\section{Materials and methods}

Cell lines and culture conditions. The trophoblast cell line HTR-8/SVneo, stored in our laboratory, was maintained in RPMI-1640 medium which was supplemented with $10 \%$ heat-inactivated FBS, $2 \mathrm{mM}$ glutamine, penicillin $(100 \mathrm{U} / \mathrm{ml})$ and streptomycin $(100 \mathrm{mg} / \mathrm{ml})$ at $37^{\circ} \mathrm{C}$ in $5 \% \mathrm{CO}_{2}$.

Plasmid construction and transfection. The ideograph of HBx protein and its truncated form is shown in Fig. 1A. The plasmid pcDNA3.1-HBx was kindly provided by Mr. Jin-Cheng Li (China Medical University, Shenyang, China). For the preparation of truncated HBx proteins, the fragments were respectively amplified from full length $\mathrm{HBx}$ by retro-transcription PCR (RT-PCR) where restriction sites were added to PCR primers (Table I) and cloned into pcDNA3.1. The sequence of the successful clone was confirmed by DNA 
Table I. Primers used to generate intact and truncated forms of HBx.

Region of HBx amplified aa $1-51$ (Intact)

aa 1-17 (Truncated 1)

aa 18-51 (Truncated 2)
Primers (5'-3')

Product (bp)

153

102

Italicized section indicates NotI restriction sites; underlined section indicates EcoRI restriction sites; F, forward; R, reverse; HBx, hepatitis B virus $\mathrm{X}$ protein.

sequencing. Transfection of plasmids to the HTR-8/SVneo cells was performed using Lipofectamine 2000 (Invitrogen, Carlsbad, CA, USA) according to the manufacturer's instructions. Briefly, the cells were plated in RPMI-1640 medium with $10 \%$ FBS and cultured until they achieved $70-80 \%$ confluency. Culture medium was then replaced with low-serum media (containing 0.5\% FBS), and then the cells were transfected with $5 \mu \mathrm{g} /$ well of the plasmid. Forty-eight hours after transfection, the pcDNA3.1-transfected (mock), pcDNA3.1-HBx-transfected (intact), pcDNA3.1-HBx- $\Delta$ C-transfected (T1), and pcDNA3.1-HBx- $\Delta \mathrm{N}$-transfected (T2) HTR-8/SVneo cells were used in the subsequent studies.

Retro-transcription PCR and quantitative real-time PCR. The expression of the relevant HBx in the established cell lines was verified by RT-PCR. Total RNA was extracted from the cell lines (the cells transfected with intact HBx, truncated 1 and 2) using TRIzol reagent (Invitrogen) according to the manufacturer's instructions. First-strand cDNAs were generated in reverse transcriptase reactions containing total RNA, poly(dT) oligonucleotides, and SuperScript II reverse transcriptase (Invitrogen). cDNAs were then subjected to RT-PCR analysis. The primers used to generate the intact and truncated forms of HBx are shown in Table I. The mRNA levels of inflammatory factors were detected using the primers that are listed in Table II. GAPDH was used as an internal control. Amplification of the primers as described above was performed with 1 cycle at $95^{\circ} \mathrm{C}$ for $10 \mathrm{~min}$ and 40 cycles of $95^{\circ} \mathrm{C}$ for $15 \mathrm{sec}$ and $60^{\circ} \mathrm{C}$ for $60 \mathrm{sec}$.

TGF- $\beta 1$ treatment. Recombinant human transforming growth factor- $\beta 1$ (TGF- $\beta 1$ ) was obtained from R\&D Systems (Minneapolis, MN, USA). The effect of TGF- $\beta 1$ on the untransfected HTR-8/SVneo and pcDNA3.1-HBx-transfected cells was determined by adding recombinant TGF- $\beta 1$ $(5 \mathrm{ng} / \mathrm{ml})$ to cell monolayers at $70 \%$ confluency. Cells were incubated for an additional $24 \mathrm{~h}$ and used in the subsequent studies.

Cell growth assay. Viability of the transfected cells was determined using the 3-(4,5-dimethylthiazolyl)-2,5-diphenyltetrazolium bromide (MTT) assay (Sigma-Aldrich, Carlsbad, CA, USA). The cells were plated in 96-well plates (1,500 cells/well) and incubated under normal culture condi-
Table II. Primers used to detect the mRNA levels of inflammatory factors.

\begin{tabular}{|c|c|}
\hline Gene & Sequence (5'-3'; forward/reverse) \\
\hline$I L-1 \alpha$ & $\begin{array}{l}\text { AGAAGAGACGGTTGAGTTTAAGCCAATCCA/ } \\
\text { ATCCAGTCGTGGAAAATCGAAGGACTC }\end{array}$ \\
\hline$I L-1 \beta$ & $\begin{array}{l}\text { CAGGGACAGGATATGGAGCAACAA/ } \\
\text { CATCTTTCAACACGCAGGACAGGT }\end{array}$ \\
\hline$T N F-\alpha$ & $\begin{array}{l}\text { AGGCCAAGCCCTGGTATGAGC/ } \\
\text { CACAGGGCAATGATCCCAAAGTAG }\end{array}$ \\
\hline$I L-6$ & $\begin{array}{l}\text { CACCCCTGACCCAACCACAAAT/ } \\
\text { TCCTTAAAGCTGCGCAGAATGAGA }\end{array}$ \\
\hline$I L-10$ & 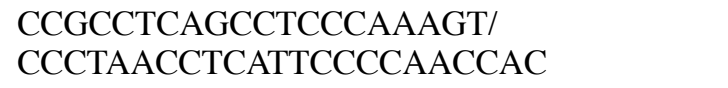 \\
\hline$I F N-\gamma$ & $\begin{array}{l}\text { TAGCAACAAAAAGAAACGAGATGACT/ } \\
\text { GATTTTGTCCCTTCGCTTTTTCC/ }\end{array}$ \\
\hline GAPDH & $\begin{array}{l}\text { TGGTATCGTGGAAGGACTCATGAC/ } \\
\text { ATGCCAGTGAGCTTCCCGTTCAGC }\end{array}$ \\
\hline
\end{tabular}

tions. After $24 \mathrm{~h}$, the cells were treated with $0.5 \mathrm{mg} / \mathrm{ml} \mathrm{MTT}$ for $4 \mathrm{~h}$ and lysed with dimethyl sulfoxide (DMSO). Absorbance rates were measured at 550-560 $\mathrm{nm}$ using a microplate reader (Bio-Rad, Hercules, CA, USA).

Apoptosis detection. Cells were trypsinized, washed twice with cold PBS, and resuspended in $200 \mu \mathrm{l}$ binding buffer. Annexin V-FITC was added to a final concentration of $0.5 \mu \mathrm{g} / \mathrm{ml}$, according to the manufacturer's instructions (Keygen, Nanjing, China). After $20 \mathrm{~min}$ of incubation at room temperature in the dark, $400 \mu \mathrm{l}$ of binding buffer containing propidium iodide (PI, $50 \mu \mathrm{g} / \mu \mathrm{l}$ ) was added, and samples were immediately analyzed on a FACSCalibur flow cytometer (Becton-Dickinson Medical Devices, Shanghai, China).

Invasion assays. Transwell chamber $(8-\mu \mathrm{m}$ pore size polycarbonate membrane; Cell Biolabs, San Diego, CA, USA) Matrigel invasion assays were performed as previously described (10). Cells $(30,000)$ were placed in the upper chamber and allowed to invade for $24 \mathrm{~h}$. Twenty fields of cells were acquired at x10 magnification and quantified. Relative invasion was expressed as a ratio to the control cells. 
$\mathbf{A}$

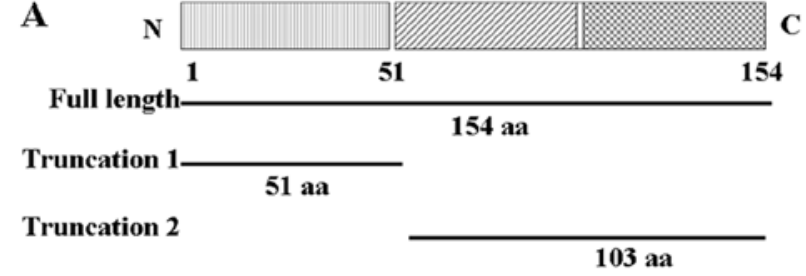

1 MAARMCCKLD PARDVLCLRP IGAESRGRPL PGPLGAVPPS SPSAVPADDG SHLSLRGLPV

61 CSFSSAGPCA LRFTSARRME TTVNAPWSLP TVLHKRTLGL SGWSMTWIEE YIKDCVFKDW 121 EELGEEIRLK VFVLGGCRHK LVCSPAPCNF FTSA

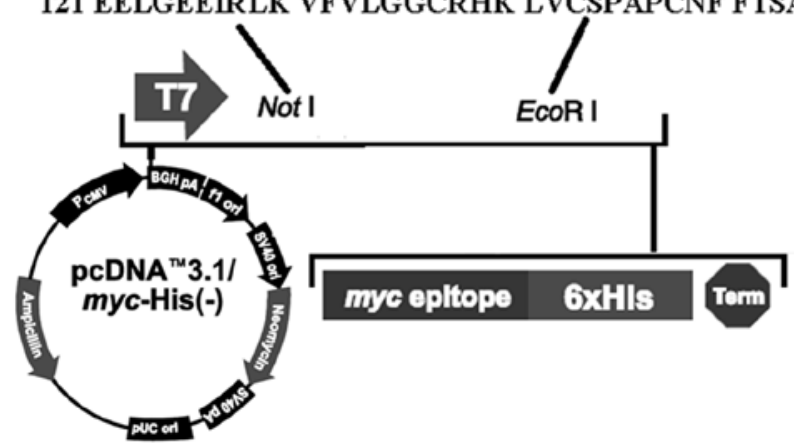

B untransfected $\mathrm{HBx} 1-462 \quad 1-153 \quad 153-462$
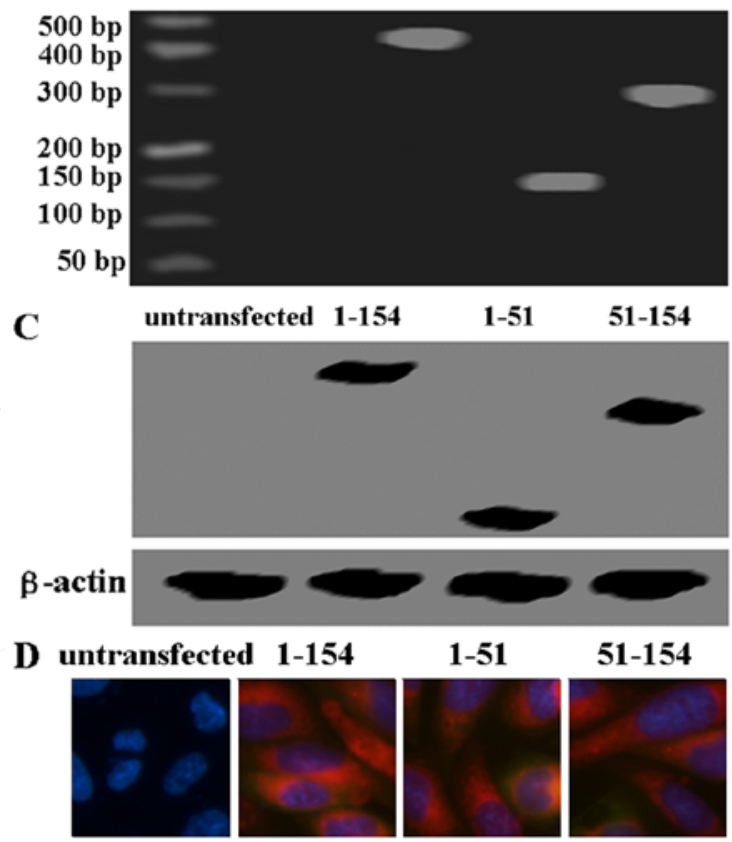

Figure 1. Detection of HBx after transfection. (A) The sequence and the truncated forms of HBx protein. (B) $H B x$ mRNA level in HTR-8/SVneo cells by RT-PCR. (C) Detection of HBx protein levels in the same set of cells by western blot analysis. $\beta$-actin was used as an internal control. (D) Detection of HBx protein levels in the same set of cells by immunofluorescence. HBx, hepatitis B virus X.

Western blotting. Cells and tissues were washed and lysed and an equal amount of proteins to ensure equal protein loading was subjected to SDS-PAGE and then blotted onto PVDF membranes (GE Healthcare Corp., Piscataway, NJ, USA). The membranes were blocked in 5\% milk-TBST and then probed with the primary antibody. TGF- $\beta 1$ RI (sc-398), p-Smad3 (sc-130218), Smad3 (sc-101154), p-Smad2 (sc-135644), Smad2 (sc-6200), E-cadherin (sc-7870), vimentin (sc-6260), $\mathrm{N}$-cadherin (sc-7939) and $\beta$-actin (sc-47778) were purchased from Santa Cruz Biotechnology (Shanghai, China). Anti-HBx (Abcam, Cambridge, UK) was used to identify the results of the transfection. $\beta$-actin was used as an internal control. The secondary antibody was anti-mouse IgG, anti-rabbit IgG, or anti-goat IgG (determined by primary antibodies) at a dilution of 1:1,000-2,000 (Amersham Biosciences, Needham, MA, USA). Then the results were detected by enhanced chemiluminescence (Amersham Pharmacia, Piscataway, NJ, USA).

Phyre database was used to generate a predicted structural model. The protein sequence of HBX was obtained from Pubmed (http://www.ncbi.nlm.nih.gov/protein/CAA49453.1) and submitted to Protein Homology/analogY Recognition Engine (Phyre version 2). Based on the homology sequence in the Phyre server, the three-dimensional structure of ING2 protein was predicted.

Physicochemical profiles of $H B X$. Physicochemical profiles, such as titration curve, hydrophobicity, antigenicity, fexibility, and solvent accessibility, were analyzed using Antheprot 5.0 software.

Statistical analysis. Statistical analyses were carried out using GraphPad Prism 5.0 (GraphPad Software Inc., San Diego, CA,
USA). Data are expressed as mean \pm standard deviation of three independent experiments performed in triplicate. Data comparisons in relation to the control were performed by one-way ANOVA. Differences were considered statistically significant if a p-value $<0.05$ was achieved.

\section{Results}

mRNA and protein levels of different HBx fragments evaluated in the cell lines. After transfection, the mRNA and protein levels of the different HBx fragments were detected using western blot analysis, RT-PCR and immunofluorescence. As shown in Fig. $1 \mathrm{~B}$ and $\mathrm{C}$, the levels of $\mathrm{HBx}$ protein and mRNA in the HTR-8/SVneo cells following transfection were higher than levels in the cells without transfection. Furthermore, intact $\mathrm{HBx}$ protein and truncated $\mathrm{HBx}$ fragments were localized in the cytoplasm by immunofluorescence assay (Fig. 1D). The results showed that intact $\mathrm{HBx}$ protein and the truncated HBx fragments were successfully transfected into the HTR-8/SVneo cells. Furthermore, 3D structures of intact HBx protein and truncated HBx fragments were predicated using Phyre version 2 (Fig. 2A). Titration curve, hydrophobicity, antigenicity, fexibility and solvent accessibility of the different HBx fragments did not have significant difference as determined using Antheprot 5.0 software (Fig. 2B and C). 2D structures of intact $\mathrm{HBx}$ protein and truncated $\mathrm{HBx}$ fragments were also confirmed (Fig. 2D).

The roles of different HBx fragments in HTR-8/SVneo cells. Cell viability was monitored using an MTT assay, and Fig. 3A shows that the proliferation rate of the HTR-8/SVneo cells was induced by both intact and truncated HBx $(\mathrm{P}<0.05)$. We utilized Annexin V-FITC and PI double staining to detect the 
A

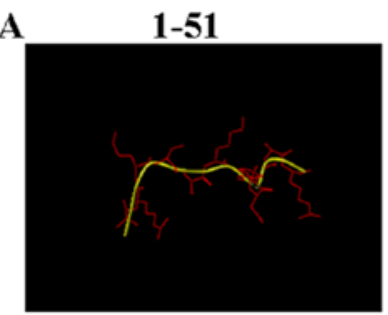

B

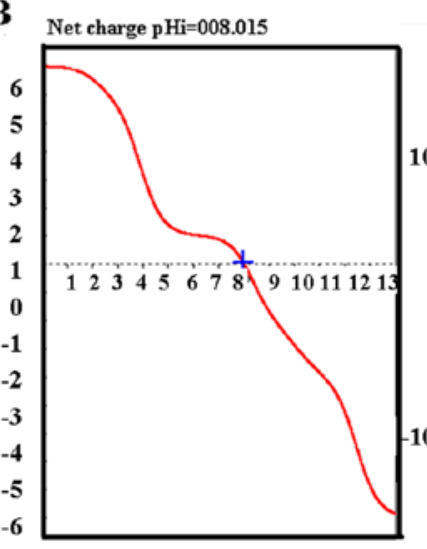

$51-154$

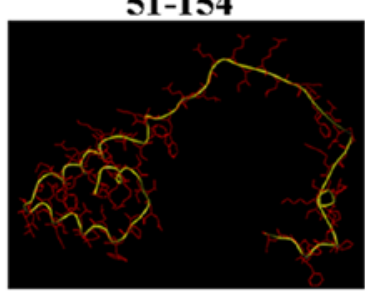

Net charge pHi=008.035

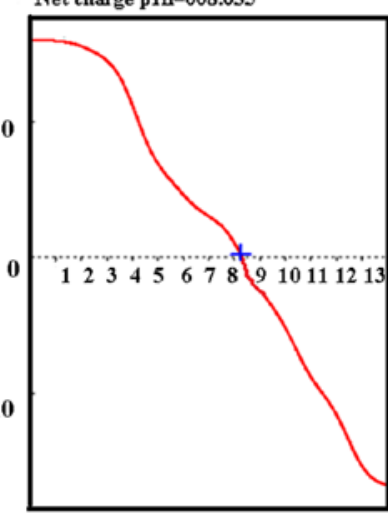

1-154

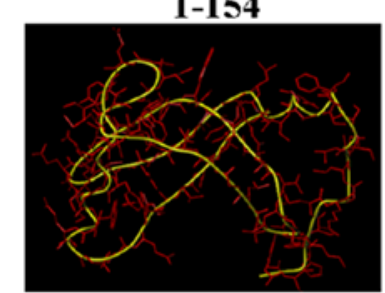

Net charge $\mathrm{pH}=008.225$

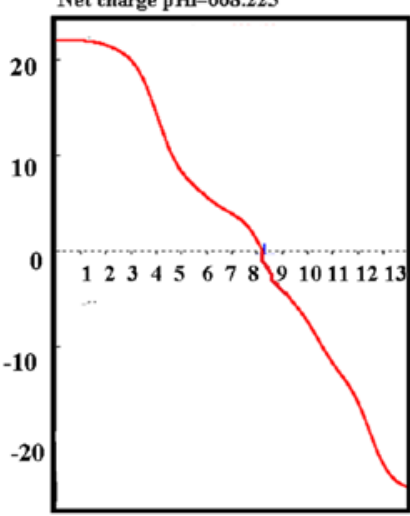

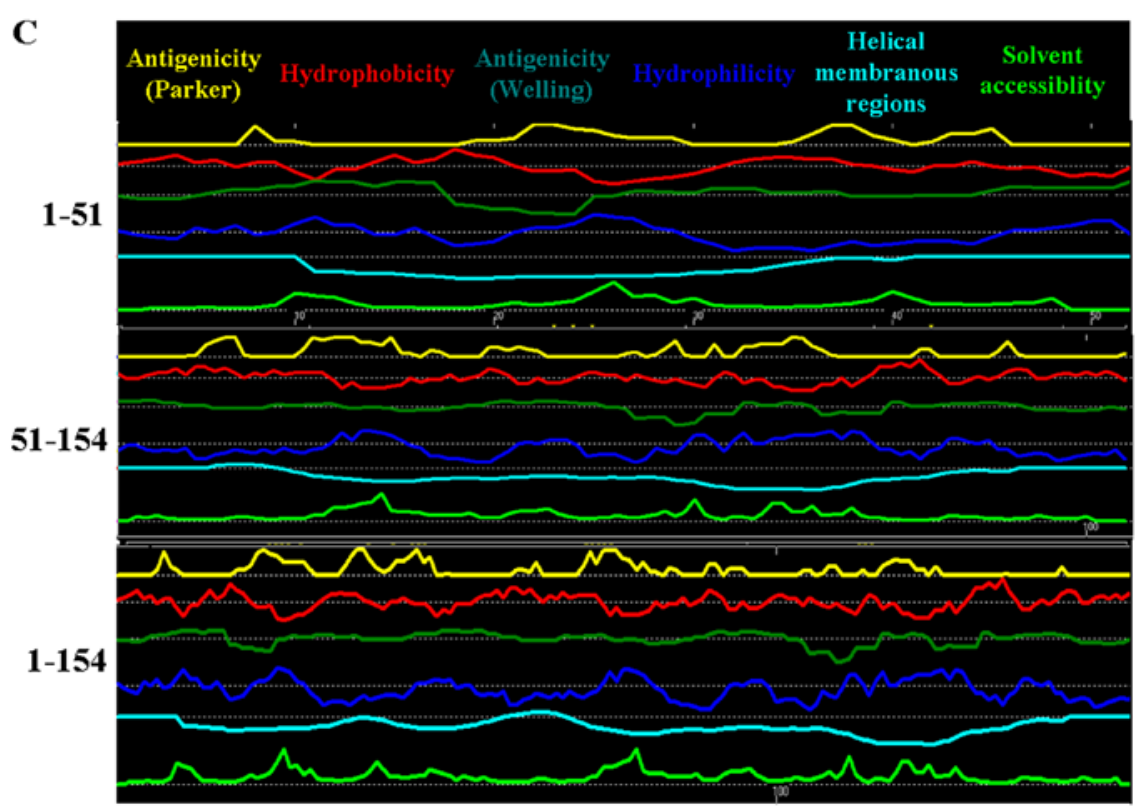

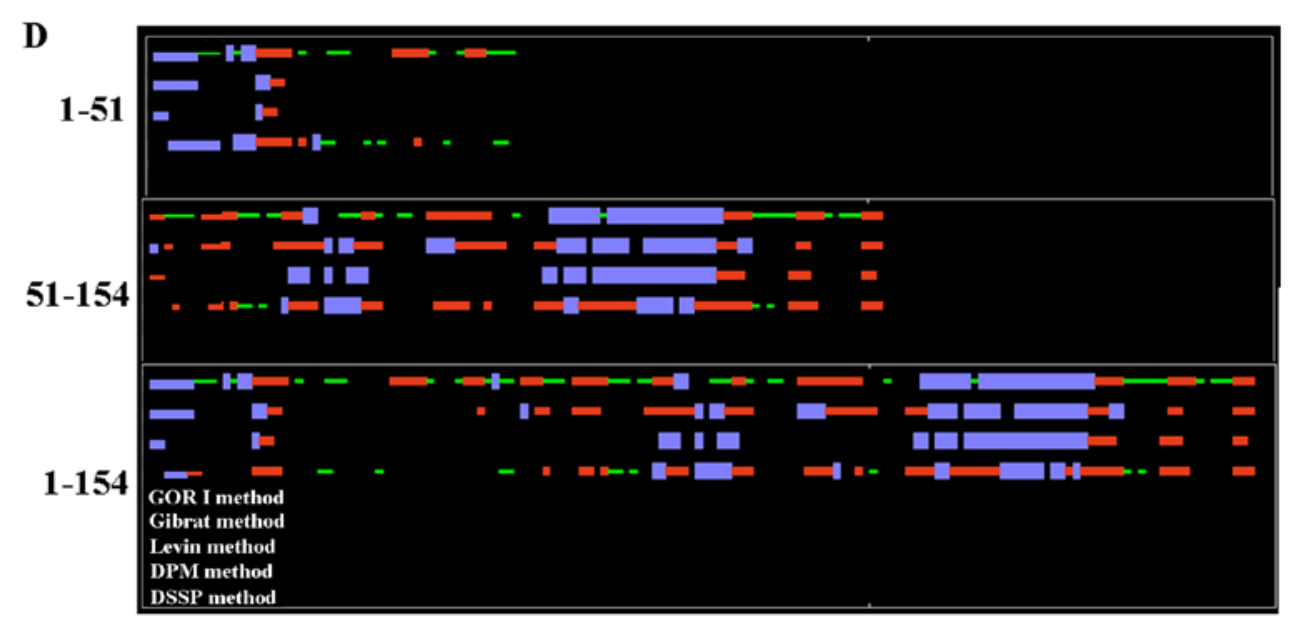

Figure 2. Physicochemical profiles of HBx and the truncated HBx fragments. (A) 3D structure of the intact HBx protein and the truncated HBx fragments. (B) Titration curves of the intact HBx protein or the truncated HBx fragments. (C) Physicochemical profiles of these three proteins. (D) 2D structure of the intact HBx protein and the truncated HBx fragments. HBx, hepatitis B virus X. 

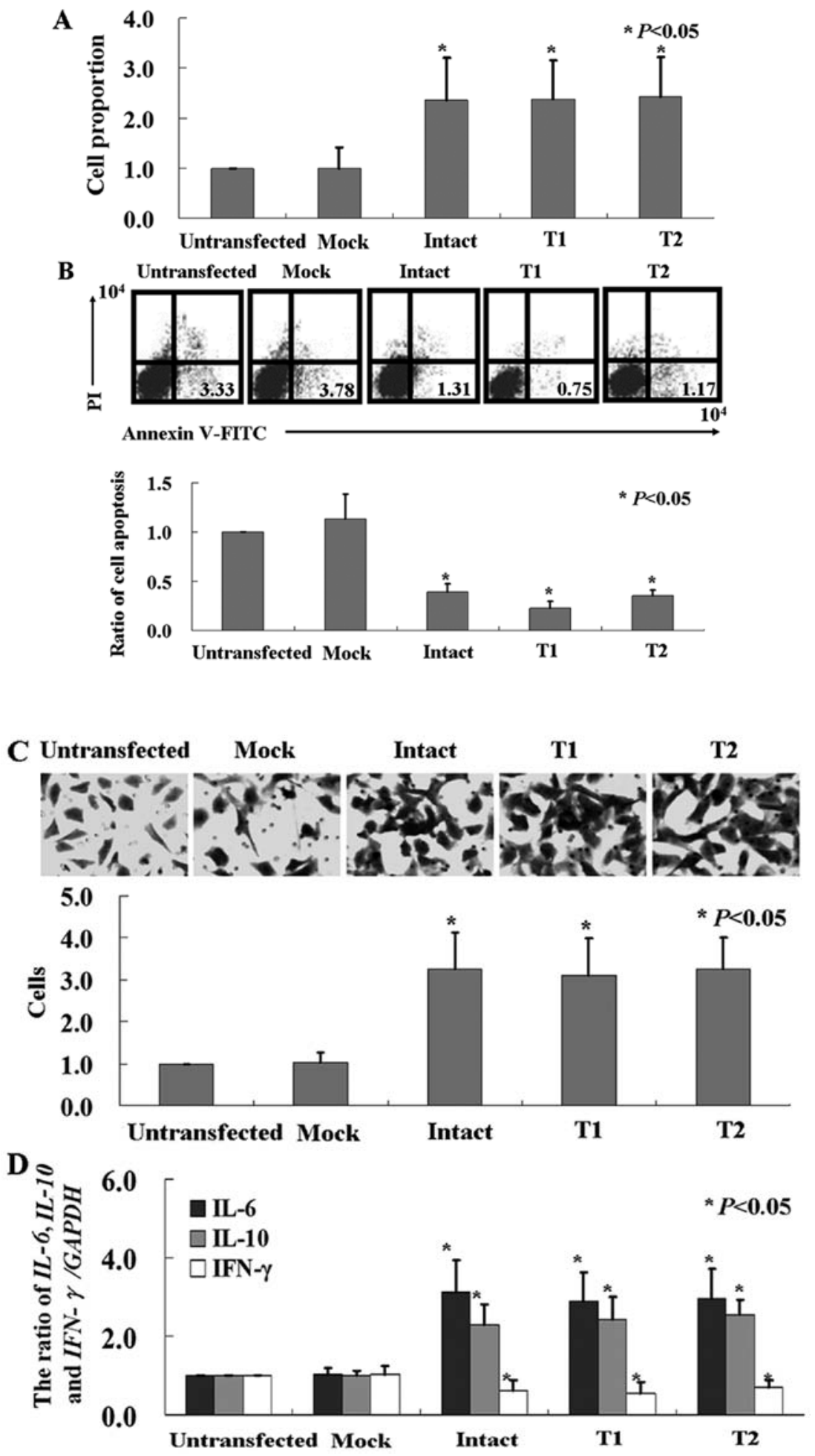

Figure 3. Biological effects of HBx on HTR-8/SVneo cells. (A) The growth inhibitory ratio of different HBx fragments on HTR-8/SVneo cells was determined by MTT assay $\left({ }^{*} \mathrm{P}<0.05\right)$. (B) Apoptotic ratio of the HBx-transfected cells was analyzed by double staining with Annexin V/PI $\left({ }^{*} \mathrm{P}<0.05\right)$. (C) Transwell assays were performed. Cells that migrated to the bottom side of the membrane were stained and counted (*P<0.05). (D) The mRNA levels of inflammatory factors $(I L-6, I L-10$ and $I F N-\gamma)$ were measured using real-time PCR ( $\mathrm{P}<0.05)$. Untransfected, untransfected HTR-8/SVneo cells; Mock, HTR-8/SVneo cells transfected with pcDNA3.1; Intact, HTR-8/SVneo cells transfected with intact HBx; T1, HTR-8/SVneo cells transfected with truncated 1; T2: HTR-8/SVneo cells transfected with truncated 2 . HBx, hepatitis B virus X; PI, propidium iodide.

apoptotic cells. Our results showed that the apoptotic ratio of the cells after transfection with the intact or truncated $\mathrm{HBx}$ was significantly decreased (Fig. 3B, P<0.05). We determined whether there were any changes in the invasive ability in the HBx-expressing cells using the Transwell assay. We found that significantly more $\mathrm{HBx}$-expressing cells migrated to the lower membrane compared with the control cells (Fig. 3C, $\mathrm{P}<0.05$ ). The mRNA levels of inflammatory factors $I L-6$ and $I L-10$ in the HBx-expressing cells were significantly higher than levels in the control cells, while $I F N-\gamma$ was lower (Fig. 3D, $\mathrm{P}<0.05$ ). 


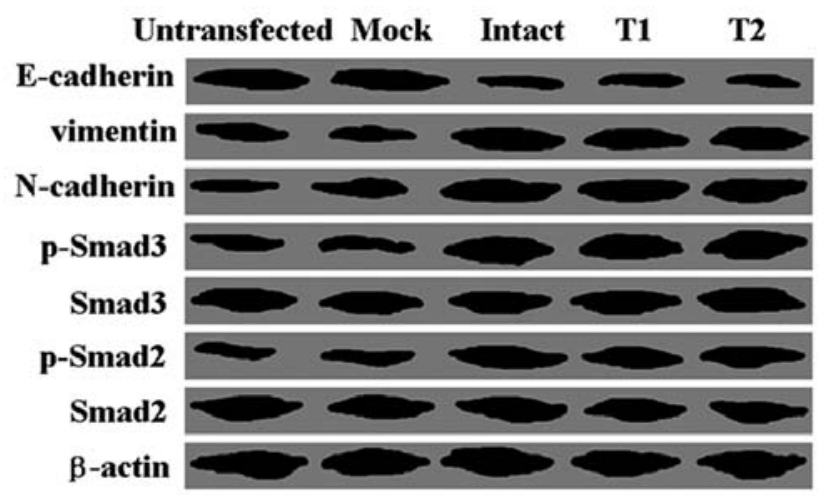

Figure 4. Western blot analysis of the Smad signaling pathway. Levels of total Smad2 and Smad3 or their phosphorylation forms in the cell lysates were determined, respectively. E-cadherin, N-cadherin and vimentin were also detected using western blot analysis. Untransfected, untransfected HTR-8/SVneo cells; Mock, HTR-8/SVneo cells transfected with pcDNA3.1; Intact, HTR-8/SVneo cells transfected with intact HBx; T1, HTR-8/SVneo cells transfected with truncated 1; T2, HTR-8/SVneo cells transfected with truncated 2. HBx, hepatitis B virus X.

In contrast, slightly increased levels of $I L-1 \alpha, I L-1 \beta$ and $T N F-\alpha$ were detected in the HTR-8/SVneo cells after HBx treatment (data not shown).

HBx fragments activate the Smad signaling pathway and induce changes in epithelial-mesenchymal transition (EMT). Furthermore, we carried out western blot analysis to identify the mechanism of apoptosis induced by HBx. We found that the levels of phospho-Smad3 and phospho-Smad2 in the transfected cells were higher than the levels in the untransfected cells, while levels of total Smad3 and Smad2 were not altered (Fig. 4). Compared with the untransfected cells, HBx-transfected cells showed a lower expression level of epithelial marker E-cadherin, while higher expression levels of mesenchymal markers vimentin and $\mathrm{N}$-cadherin were noted (Fig. 4).

TGF- $\beta 1$ decreases HTR-8/SVneo cell proliferation and invasion, while increases HBx-transfected HTR-8/SVneo cell proliferation and invasion. TGF- $\beta 1$ was used to further clarify the effect of the Smad signaling pathway on HTR-8/ SVneo cells. HTR-8/SVneo cells were treated with recombinant human TGF- $\beta 1(5 \mathrm{ng} / \mathrm{ml})$. The proliferation rate of the HTR-8/SVneo cells was inhibited by recombinant human TGF- $\beta 1$ (Fig. 5A, P<0.05). Annexin V-FITC and PI double staining showed that recombinant human TGF- $\beta 1$ induced apoptosis in the HTR-8/SVneo cells (Fig. 5B, P<0.05). Matrigel invasion assay showed that treatment with $5 \mathrm{ng} / \mathrm{ml}$ of TGF- $\beta 1$ significantly decreased HTR-8/SVneo cell invasion (Fig. 5C, $\mathrm{P}<0.05$ ). The mRNA levels of inflammatory factors $I L-6$ and $I L-10$ in the HTR-8/SVneo cells treated with TGF- $\beta 1$ were lower than levels in the control cells (Fig. 5D, $\mathrm{P}<0.05)$. Western blot analysis showed that TGF- $\beta 1$ treatment induced TGF- $\beta 1$ RI expression, and Smad 2 and Smad3 phosphorylation in the HTR-8/SVneo cells (Fig. 5E). Although TGF- $\beta 1$ also induced Smad 2 and Smad3 phosphorylation in the HBX-transfected HTR-8/SVneo cells (Fig. 5E), opposite effects of TGF- $\beta 1$ on the HBx-transfected HTR-8/SVneo cells were observed when compared with the untransfected cells (Fig. 5). TGF- $\beta 1$ increased proliferation (Fig. $5 \mathrm{~A}, \mathrm{P}<0.05)$ and invasion (Fig. 5C, $\mathrm{P}<0.05$ ), inhibited apoptosis (Fig. 5B, $\mathrm{P}<0.05$ ), and induced the inflammatory response (Fig. 5D, $\mathrm{P}<0.05)$ in the HBx-transfected HTR-8/SVneo cells.

\section{Discussion}

Mother-to-infant transmission of HBV can occur as an intrauterine, intrapartum or postpartum infection (4). Intrauterine infection by HBV is mainly transmitted through the placenta. HBV can reach the placenta though maternal blood and infect placental trophoblast cells and then survive and replicate in the cells, and functional protein HBxAg is produced (11).

In the present study, we confirmed that HBx inhibited apoptosis and increased invasive ability in the HTR-8/ SVneo cells. Bai et al (11) found that the apoptosis index of $\mathrm{HBV}$-infected high replication cells was lower than that of uninfected ones. Liu et al (12) found that HBx could promote hepatoma cell invasion and metastasis. The HBx genome consists of an $\mathrm{N}$-terminal negative regulatory domain and a C-terminal transactivation domain (13). The HBx fragment at the C-terminal can strongly enhance the proliferation and growth of liver cells (14). However, Chau et al (15) found that the first 50 aa $\mathrm{NH}_{2}$ region of $\mathrm{HBx}$ has the ability to resist cell death stimulation. Notably, we found that two truncated forms of HBx (N-terminal or C-terminal mutants) had similar effects as intact HBx on the HTR-8/SVneo cells. Based on our present data, we could not explain these contradictory results of $\mathrm{HBx}$ in different cells as $\mathrm{HBx}$ has been reported to be both pro-apoptotic and anti-apoptotic. We will investigate the contradiction in future research.

The trophoblast is a cell type with endocrine functions that also secretes various cytokines throughout the gestation period (16). IL-10 was found to be a critical molecule for successful pregnancy outcome in both human and mouse pregnancy models (17). Lack of IL-10 expression in trophoblasts may contribute to an increased inflammatory response in the placenta (18). Regulation of IL-6 secretion during pregnancy is essential for maintaining normal gestation. Elevated IL-6 is observed during recurrent miscarriage, pre-eclampsia and preterm delivery (19). Moreover, IFN- $\gamma$ can be deleterious to pregnancy, as determined by in vivo animal model studies (20). Hu et al (21) provided direct evidence that IFN- $\gamma$ can influence 

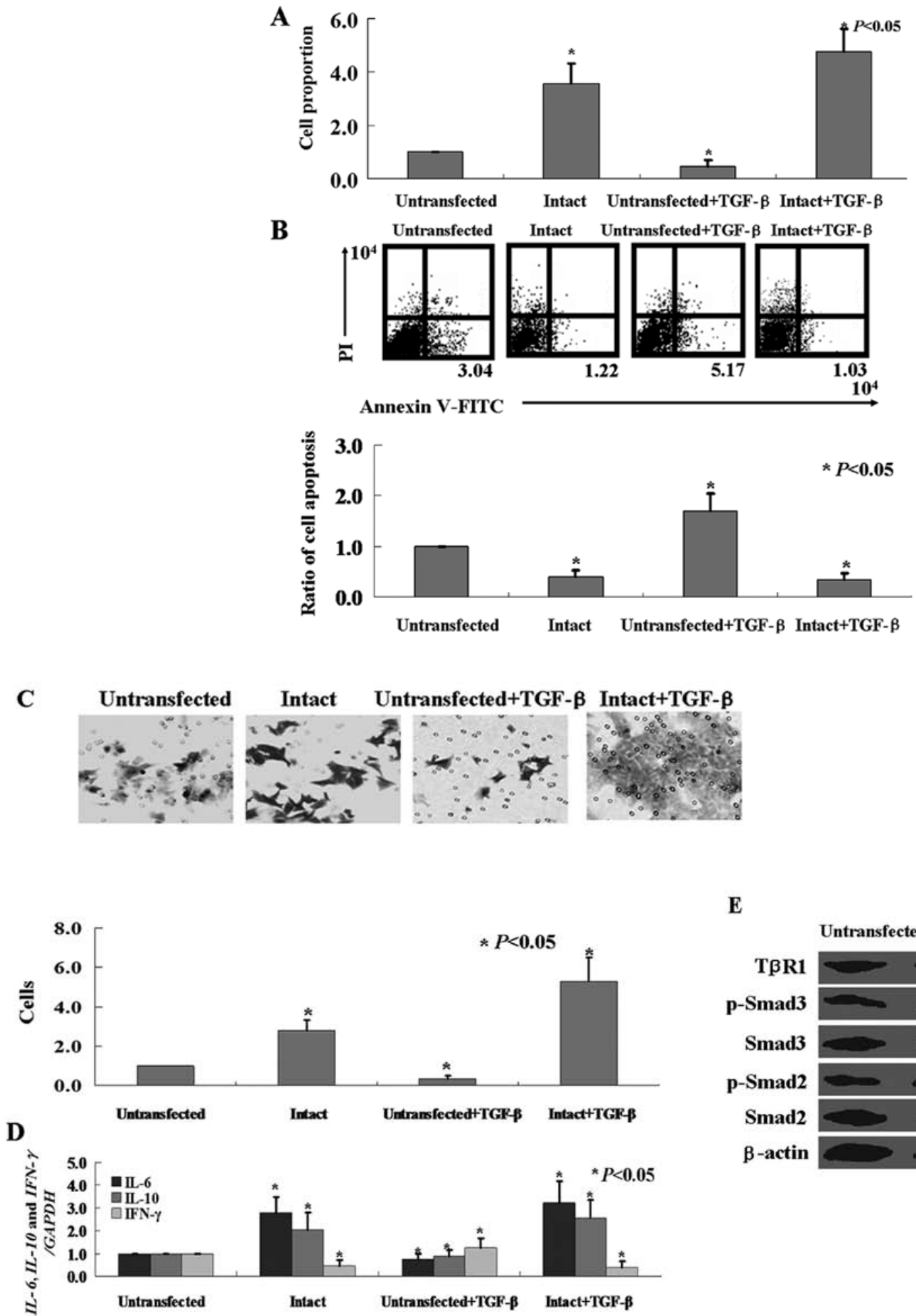

$\mathbf{E}$

TGF- $\beta(5 \mathrm{ng} / \mathrm{ml})$ Untransfected Intact Untransfected Intact

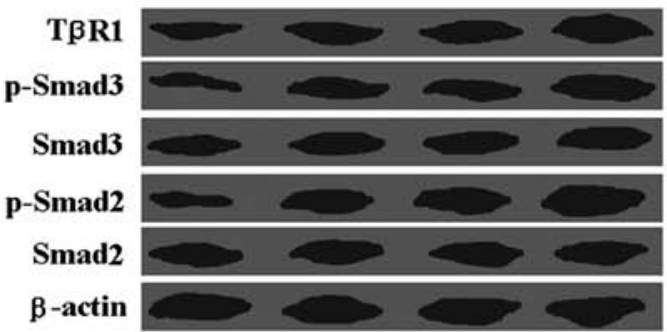

Figure 5. Biological effects of TGF- $\beta 1$ on HTR-8/SVneo cells with or without HBx transfection. (A) The growth inhibitory ratio was determined by MTT assay. (B) Apoptotic ratio was analyzed by double staining with Annexin V/PI. (C) Transwell assays were performed to detect the invasion of cells. (D) The mRNA levels of inflammatory factors $(I L-6, I L-10$ and $I F N-\gamma)$ were measured using real-time PCR. (E) Western blot analysis of the Smad signaling pathway. Untransfected, untransfected HTR-8/SVneo cells; Intact, HTR-8/SVneo cells transfected with intact HBx; Untransfected+TGF- $\beta 1$, untransfected HTR-8/ SVneo cells were treated with TGF- $\beta 1$ ( $5 \mathrm{ng} / \mathrm{ml})$; Intact+TGF- $\beta 1$, HTR-8/SVneo cells transfected with intact HBx were treated with TGF- $\beta 1$ (5 ng/ml). HBx, hepatitis B virus X; PI, propidium iodide.

extravillous cytotrophoblast (EVT) outgrowth and migration. In the present study, we also found upregulation of $I L-6$ and $I L-10$ mRNA levels and downregulation of the $I F N-\gamma$ mRNA level in the HTR-8/SVneo cells following HBx transfection.
EMT is a process whereby epithelial cells change to a mesenchymal phenotype, and this process is important in the progression of human carcinomas to a more invasive, metastatic capacity (22). We confirmed that HBx-induced 


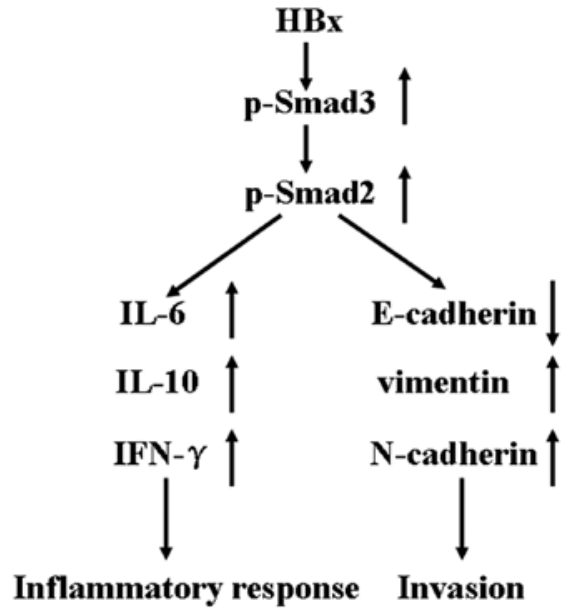

Figure 6. Schematic model of the mechanism proposed for HBx in HTR-8/SVneo cells. HBx, hepatitis B virus X.

invasion of HTR-8/SVneo cells involves EMT. Furthermore, we found that the Smad signaling pathway was activated by HBx in the HTR-8/SVneo cells. The Smad family of proteins (Smad1-8) are classified according to their different functions; Smad1, 2, 3, 5 and 8 are 'receptor-regulated SMADs', Smad4 is termed the 'common-mediator SMAD', and Smad6 and 7 are 'inhibitory SMADs' (23). These proteins regulate numerous cellular processes, such as cell proliferation, differentiation, apoptosis and control of developmental fate (24). Robust activation of Smad2 and Smad3 is through phosphorylation of C-terminal regulatory residues (23). In the present study, we confirmed that $\mathrm{p}$-Smad 2 and $\mathrm{p}$-Smad 3 were significantly upregulated in the HTR-8/SVneo cells following HBx transfection. To confirm the effect of the Smad signaling pathway on HTR-8/SVneo cells, TGF- $\beta 1$ was used as a control. TGF- $\beta 1$ activates TGF- $\beta$ receptor I (T $\beta R I)$ and T $\beta$ RII, which results in the phosphorylation of receptor-regulated SMAD2/3 proteins (25). In the present study, we found that TGF- $\beta 1$ treatment induced proliferation and invasion, and inhibited apoptosis in the HTR-8/SVneo cells via activation of the Smad signaling pathway. Cheng et al (26) also found that TGF- $\beta 1$ induced the downregulation of VE-cadherin and decreased cell invasion in human trophoblast cells. Notably, we found that activation of the Smad signaling pathway had opposite roles in the HTR-8/SVneo cells with HBx transfection. HBx plays critical roles in the pathogenesis of hepatocellular carcinoma development, based on its tumorigenic activity in vitro and in vivo (27). According to the results of previous studies, we concluded that HTR-8/SVneo cells with HBx transfection also underwent cancerous transformation. More evidence was provided by Murata et al (28). They found that HBx shifts TGF- $\beta$ signaling from tumor suppression to oncogenesis in early chronic hepatitis B. However, the mechanism in HTR-8/ SVneo cells should be validated in future studies.

Taken together, in all our experiments, we elucidated the biological effects of different $\mathrm{HBx}$ fragments on HTR-8/SVneo cells. However, there was no difference among these fragments. We demonstrated that HBx activates the Smad signaling pathway in HTR-8/SVneo cells. After the signaling pathway was activated, a lower apoptotic ratio, higher cell motility, and an enhanced inflammatory response were observed in the HTR-8/SVneo cells (Fig. 6). In further studies, we will truncate other domains of $\mathrm{HBx}$ and detect their activities.

\section{Acknowledgements}

We thank Mr. Jin-Cheng Li (China Medical University, Shenyang, China) for providing the plasmid pcDNA3.1-HBx.

\section{References}

1. Lok AS: Chronic hepatitis B. N Engl J Med 346: 1682-1683, 2002.

2. Xu DZ, Yan YP, Choi BC, Xu JQ, Men K, Zhang JX, Liu ZH and Wang FS: Risk factors and mechanism of transplacental transmission of hepatitis B virus: A case-control study. J Med Virol 67: 20-26, 2002.

3. Zhang SL, Yue YF, Bai GQ, Shi L and Jiang H: Mechanism of intrauterine infection of hepatitis $B$ virus. World $J$ Gastroenterol 10: 437-438, 2004.

4. Ohto H, Lin HH, Kawana T, Etoh T and Tohyama H: Intrauterine transmission of hepatitis B virus is closely related to placental leakage. J Med Virol 21: 1-6, 1987.

5. Veerbeek JH, Nikkels PG, Torrance HL, Gravesteijn J, Post Uiterweer ED, Derks JB, Koenen SV, Visser GH, Van Rijn BB and Franx A: Placental pathology in early intrauterine growth restriction associated with maternal hypertension. Placenta 35: 696-701, 2014

6. Lunghi L, Ferretti ME, Medici S, Biondi C and Vesce F: Control of human trophoblast function. Reprod Biol Endocrinol 5: 6-20, 2007.

7. Bouchard MJ and Schneider RJ: The enigmatic X gene of hepatitis B virus. J Virol 78: 12725-12734, 2004.

8. Tang H, Oishi N, Kaneko S and Murakami S: Molecular functions and biological roles of hepatitis B virus x protein. Cancer Sci 97: 977-983, 2006.

9. Zhang H, Shan CL, Li N, Zhang X, Zhang XZ, Xu FQ, Zhang S, Qiu LY, Ye LH and Zhang XD: Identification of a natural mutant of HBV X protein truncated 27 amino acids at the $\mathrm{COOH}$ terminal and its effect on liver cell proliferation. Acta Pharmacol Sin 29: 473-480, 2008.

10. Liu S, Cui H, Li Q, Zhang L, Na Q and Liu C: RhoGDI2 is expressed in human trophoblasts and involved in their migration by inhibiting the activation of RAC1. Biol Reprod 90: 88, 2014.

11. Bai G, Wang Y, Zhang L, Tang Y and Fu F: The study on the role of hepatitis B virus $X$ protein and apoptosis in HBV intrauterine infection. Arch Gynecol Obstet 285: 943-949, 2012.

12. Liu H, Xu L, He H, Zhu Y, Liu J, Wang S, Chen L, Wu Q, Xu J and $\mathrm{Gu}$ J: Hepatitis $\mathrm{B}$ virus $\mathrm{X}$ protein promotes hepatoma cell invasion and metastasis by stabilizing Snail protein. Cancer Sci 103: 2072-2081, 2012.

13. Luo N, Cai Y, Zhang J, Tang W, Slagle BL, Wu X and He S: The $\mathrm{C}$-terminal region of the hepatitis $\mathrm{B}$ virus $\mathrm{X}$ protein is required for its stimulation of HBV replication in primary mouse hepatocytes. Virus Res 165: 170-178, 2012.

14. Wang Q, Zhang W, Liu Q, Zhang X, Lv N, Ye L and Zhang X: A mutant of hepatitis B virus X protein (HBxDelta127) promotes cell growth through a positive feedback loop involving 5-lipoxygenase and fatty acid synthase. Neoplasia 12: 103-115, 2010.

15. Chau DK, Chen GG, Zhang H, Leung BC, Chun S and Lai PB: Differential functions of $\mathrm{C}$ - and $\mathrm{N}$-terminal hepatitis $\mathrm{B} \times$ protein in liver cells treated with doxorubicin in normoxic or hypoxic condition. PLoS One 7: e50118, 2012.

16. Noyola-Martínez N, Díaz L, Avila E, Halhali A, Larrea F and Barrera D: Calcitriol downregulates TNF- $\alpha$ and IL-6 expression in cultured placental cells from preeclamptic women. Cytokine 61: 245-250, 2013.

17. Sharma S, Stabila J, Pietras L, Singh AR, McGonnigal B, Ernerudh J, Matthiesen L and Padbury JF: Haplotype-dependent differential activation of the human IL-10 gene promoter in macrophages and trophoblasts: Implications for placental IL-10 deficiency and pregnancy complications. Am J Reprod Immunol 64: 179-187, 2010.

18. Dong Q, Fan R, Zhao S and Wang Y: Over-expression of SOCS-3 gene promotes IL-10 production by JEG-3 trophoblast cells. Placenta 30: 11-14, 2009. 
19. Prins JR, Gomez-Lopez N and Robertson SA: Interleukin-6 in pregnancy and gestational disorders. J Reprod Immunol 95: 1-14, 2012.

20. Liu Z, Chen Y, Yang Y and Peng JP: The effect on MHC class II expression and apoptosis in placenta by IFNgamma administration. Contraception 65: 177-184, 2002.

21. Hu Y, Tan R, MacCalman CD, Eastabrook G, Park SH, Dutz JP and von Dadelszen P: IFN-gamma-mediated extravillous trophoblast outgrowth inhibition in first trimester explant culture: A role for insulin-like growth factors. Mol Hum Reprod 14: 281-289, 2008.

22. Thomson S, Petti F, Sujka-Kwok I, Mercado P, Bean J, Monaghan M, Seymour SL, Argast GM, Epstein DM and Haley JD: A systems view of epithelial-mesenchymal transition signaling states. Clin Exp Metastasis 28: 137-155, 2011.

23. Schmierer B and Hill CS: TGFbeta-SMAD signal transduction: Molecular specificity and functional flexibility. Nat Rev Mol Cell Biol 8: 970-982, 2007.

24. Weiss A and Attisano L: The TGFbeta superfamily signaling pathway. Wiley Interdiscip Rev Dev Biol 2: 47-63, 2013.
25. Busnadiego O, González-Santamaría J, Lagares D Guinea-Viniegra J, Pichol-Thievend C, Muller L and Rodríguez-Pascual F: LOXL4 is induced by transforming growth factor $\beta 1$ through Smad and JunB/Fra2 and contributes to vascular matrix remodeling. Mol Cell Biol 33: 2388-2401, 2013.

26. Cheng JC, Chang HM and Leung PC: Transforming growth factor- $\beta 1$ inhibits trophoblast cell invasion by inducing Snail-mediated down-regulation of vascular endothelial-cadherin protein. J Biol Chem 288: 33181-33192, 2013.

27. Xu Z, Yen TS, Wu L, Madden CR, Tan W, Slagle BL and Ou JH: Enhancement of hepatitis B virus replication by its X protein in transgenic mice. J Virol 76: 2579-2584, 2002.

28. Murata M, Matsuzaki K, Yoshida K, Sekimoto G, Tahashi Y, Mori S, Uemura Y, Sakaida N, Fujisawa J, Seki T, et al: Hepatitis B virus $X$ protein shifts human hepatic transforming growth factor (TGF)-beta signaling from tumor suppression to oncogenesis in early chronic hepatitis B. Hepatology 49: 1203-1217, 2009. 